\title{
Editor-in-Chief's Perspective
}

On behalf of my colleagues on the Editorial Board, let me inform you about the aims and the developments of our journal. We launched Thalamus \& Related Systems in April 2001 because thalamic research has entered a new era in which fine morphological investigations lead to a level of resolution that was hitherto unexpected, in vitro and in vivo electrophysiological studies contribute in concert to a better understanding of intrinsic neuronal properties and complex network operations underlying normal behavioral states and seizures, computational studies on realistic models succeed in predicting new mechanisms that are further testable experimentally, and combined physiological and clinical investigations provide methods to localize and treat various pathological syndromes.

Despite the existence of numerous periodicals in neuroscience, we have received a steady stream of excellent articles submitted for publication from a variety of morphological, physiological, computational, and clinical fields, all centered on the thalamic world and related structures. Thus, in the morphological/immunohistochemical domain we have published highly original articles dealing with the delineation of thalamic nuclei based on immunoreactivity for calcium-binding proteins, thalamocortical projections in primates, and the circuits between thalamus and basal ganglia. In vitro and in vivo electrophysiological studies have reported new data on the role of intrinsic currents of thalamic neurons, the invasion of distal dendrites by action potentials and sensory-evoked EPSPs, thalamocortical activation patterns using voltage-sensitive dye imaging, and thalamic plasticity impacting on cortical processes. Computational studies proposed firing-rate models of spike-frequency adaptation in thalamic cells, analyzed the possible mechanisms of deep brain stimulation and, more recently, explored the variability of quantal synaptic currents in thalamocortical cells. Last but not least, clinical studies exposed data regarding the effects of thalamic and subthalamic stimulation in different forms of seizures and resting tremor in Parkinson's disease. A special section in the November 2001 issue was devoted to the application of brain stimulation and the clinical/neurosurgical aspects of thalamocortical dysrhythmia. All the above shows the large range of topics and the interconnections between fields of interest that would otherwise seem disparate.

The time from submission of papers to editorial decision was very short-2-3 weeks. The staff at Elsevier processed the manuscripts promptly and efficiently in sending page proofs and finally to print.

In the future, we will continue to combine publications of papers belonging to different fields with special issues focused on one topic, including an upcoming issue on Pain whose guest editors will be E.G. Jones and W.D. Willis, Jr. Other proposals for such special issues are encouraged together with feature articles and reviews on different aspects on the thalamus and its relations with cerebral cortex, basal ganglia and brainstem.

Thalamus \& Related Systems is now available in over 400 institutions, is highlighted in the new Elsevier Neuroscience catalogue and on Neuroscion (http://neuroscion.com) as well as on ScienceDirect. Recently, we have implemented electronic submission that will function together with the classical submission of papers with hard copies of text and figures. The success of a journal depends on submission of high quality papers. On behalf of the editorial board of Thalamus \& Related Systems, I invite you to submit papers on recent findings, reviews and perspectives on topics related to the complex functions of the thalamus and related structures.

M. Steriade

Laboratory of Neurophysiology Faculty of Medicine, Laval University Quebec, Que., Canada GIK 7P4 Tel.: +1-418-656-5547; fax: +1-418-656-3236 E-mail address: mircea.steriade@phs.ulaval.ca

(M. Steriade) 